\title{
Determining the parameters of ionospheric perturbation caused by earthquakes using the quasi-optimum algorithm of spatiotemporal processing of TEC measurements
}

\author{
Vladislav V. Kiryushkin and Edward L. Afraimovich \\ Institute of Solar-Terrestrial Physics SB RAS, P.O. box 4026, Irkutsk, 664033, Russia \\ (Received June 27, 2006; Revised November 15, 2006; Accepted December 11, 2006; Online published May 7, 2007)
}

\begin{abstract}
We present the quasi-optimum algorithm of localization of a source of coseismic ionospheric perturbation based on GPS receivers' network data processing. The initial data of the algorithm are the series of variations of ionospheric total electron content (TEC), reconstructed using the measurements of the phase delays of GPS signals. In order to select the TEC increments caused by ionospheric perturbation due to the earthquake, the TEC series are filtered using a special procedure. The algorithm realizes coherent summation of all TEC series of GPS array reasoning from the maximization of the energy of the total signal of the ionospheric response to earthquake. The quasi-optimum algorithm allows determination of the perturbation propagation velocity as well as of the coordinates, height and "switch-on" time of a source of coseismic ionospheric disturbance without prior information about the perturbation form and the site and time of the main shock of earthquake. We used the algorithm for measuring the parameters of ionospheric perturbations which accompanied the earthquake in the vicinity of Hokkaido Island on September 25, 2003 and the earthquake near the south coast of Honshu Island on September 5, 2004. The results of these experiments show the high accuracy of the perturbation source coordinates estimation $(33 \mathrm{~km}$ and $27 \mathrm{~km}$ respectively with reference of the earthquakes epicenters). The estimations of perturbations propagation velocity $(820 \pm 60 \mathrm{~m} / \mathrm{s}$ and $460 \pm 40 \mathrm{~m} / \mathrm{s})$, heights $(340 \pm 80 \mathrm{~km}$ and $370 \pm 130 \mathrm{~km}$ ) and "switch-on" delay (346 s and $507 \mathrm{~s}$ ) of a source of the perturbation obtained in both experiments are in agreement with a theory according to which coseismic atmospheric disturbance propagates within a narrow cone of zenith angles up to ionospheric heights and then diverges in the form of a spherical wave with the radial velocity close to the speed of sound at these heights. It is also in agreement with the results of earlier researches.
\end{abstract}

Key words: GPS, earthquakes, total electron content, arrays, spatiotemporal processing.

\section{Introduction}

The method of Global Positioning System (GPS) monitoring of the ionosphere is currently widely used for detecting and studying ionospheric disturbances of anthropogenic and natural origins. Anthropogenic sources include nuclear events, explosions, rocket launching and powerful radio emission, while natural sources are solar flares, earthquakes, volcanoes, heavy thunderstorms and auroral heating. The development of regional and international networks of densely spaced and continuously recording GPS stations together with high accuracy of phase measurements in the GPS system provides an opportunity to investigate such ionospheric perturbations with high spatial and temporal resolution. Using GPS receivers, Calais and Minster (1995) 10-30 min after the earthquake in California on 17 January 1994, observed in the time series of total electron content (TEC) anomalous signal in the period range 3-10 min. The frequency and propagation phase velocity $(300-600 \mathrm{~m} / \mathrm{s})$ agreed with the results of numerical simulation for atmospheric gravity waves (AGW) caused by a

Copyright (c) The Society of Geomagnetism and Earth, Planetary and Space Sciences (SGEPSS); The Seismological Society of Japan; The Volcanological Society of Japan; The Geodetic Society of Japan; The Japanese Society for Planetary Sciences; TERRAPUB. rapid uplift of subsidence of the Earth' surface during the earthquake. The velocity value was determined as a ratio of the sub-ionospheric point (SIP) distance from the epicenter to the time delay of perturbation arrival to the corresponding SIP.

Calais and Minster (1996) detected ionospheric perturbation following the STS-58 Space Shuttle launch that lasted for $35 \mathrm{~min}$, with periods of less $10 \mathrm{~min}$. They interpreted this perturbation as a direct propagation of the front of the shock-acoustic wave (SAW) caused by the launch. Five GPS receivers were installed while conducting three powerful explosions in eastern Wyoming (USA) in July and August 1996 (Calais et al., 1998). Ionospheric disturbances appeared 10-15 min after the explosion and lasted about $30 \mathrm{~min}$, propagated with a horizontal velocity $1200 \mathrm{~m} / \mathrm{s}$. The velocity value was determined to be the ratio of difference of receivers' distances from the explosion epicenter to the difference of arrival time of the ionospheric perturbation registered by these receivers. Fitzgerald (1997), $565 \mathrm{~s}$ after the surface explosion in New Mexico, detected the ionospheric TEC disturbance with an amplitude of 0.14 TECU (total electron content unit, 1 TECU $=10^{16} \mathrm{~m}^{-2}$ ) and duration of $80 \mathrm{~s}$. The acoustic disturbance needed to produce such ionospheric perturbation was well modeled as an $\mathrm{N}$ - 
shape wave with the spatial dimensions and relative amplitude of $35 \mathrm{~km}$ and $12 \%$, respectively, propagating with a radial velocity $700 \mathrm{~m} / \mathrm{s}$.

Afraimovich et al. (2001a) proposed the interferometric method for determining the parameters (including angular characteristics of the wave vector and phase velocity) of SAW generated during rocket launching. The authors used GPS-arrays of three sites, the distance between which did not exceed about one-half the wavelength of the perturbation. This method provided an estimation of SAW parameters without a priori information about the site and time of a rocket launching. They found SAW period 270-360 s and elevation angle of SAW wave vector $30^{\circ}-60^{\circ}$. The obtained value of SAW phase velocity (900-1200 m/s) approached the sound velocity at the ionospheric heights. The method (Afraimovich et al., 2001a) was also used for investigating SAW caused by earthquakes (Afraimovich et al., 2001b).

Afraimovich (2000) was the first to suggest the concept of a new technology for the detection of ionospheric disturbances using GPS arrays. According this concept the TEC measurements along the GPS line-of-sight (LOS) were considered to be elements of nonequidistant phased antenna arrays of the acoustic detectors located at the ionospheric heights. The TEC measurements were subjected to processing using spatiotemporal analysis algorithms. On the basis of this concept the optimum method of spatiotemporal processing of TEC series was developed (Afraimovich et al., 2001c). The method enabled the phase velocity and arrival direction of ionospheric perturbation to be determined (on the assumption that wave front of a disturbance is plane). The proposed method was used for studying the ionospheric perturbations caused by SAW generated during rockets launching.

Afraimovich et al. (2002) proposed that the method be used for determining the spatial-temporal characteristics of ionospheric perturbation recorded in the near-field region of the earthquake epicenter. The method was based on the optimum spatiotemporal processing of TEC series and provided an estimation of the phase velocity and source location of ionospheric disturbance in the spherical wavefront approximation. The efficiency of the proposed method was estimated by analyzing the TEC variations observed during the June 4, 2000, South Sumatra earthquake.

In order to investigate the properties of ionospheric perturbation recorded by the Southern California Integrated GPS Network (SCIGN) Calais et al. (2003) applied array processing techniques derived from seismic data analysis. The authors assumed that the perturbations propagate as plane-waves. They varied the perturbation velocity and azimuth values. For each velocity and azimuth pair, they calculated the maximum amplitude of coherent sum of all TEC series of the GPS array. The largest maximum amplitude corresponded to the best-fit propagation velocity and direction. The authors found perturbation propagation velocities $500 \mathrm{~m} / \mathrm{s}, 600 \mathrm{~m} / \mathrm{s}$, and $650 \mathrm{~m} / \mathrm{s}$ and propagation azimuth N45E, N70W, and N100E, respectively.

Heki and Ping (2005) investigated coseismic ionospheric perturbation using data of the dense GPS array GEONET (GPS Earth Observation Network). In order to determine the propagation velocity they plotted the "travel time dia- gram". These authors determined the propagation velocity value as the slope of the best-fit line of the first peak arrival times. To visualize the perturbation propagation directivity, Heki and Ping plotted on the map snapshots of wavelettransformed TEC values. For the Tokachi earthquake, the obtained values of perturbation propagation velocity and azimuth were $1250 \mathrm{~m} / \mathrm{s}$ and $230^{\circ}-280^{\circ}$, respectively.

The method (Afraimovich et al., 2002) was subsequently refined (Afraimovich et al., 2005, 2006). After updating, the methods provided an estimation of the phase velocity and source location of ionospheric disturbance as well as the height and "switch-on" time of the perturbation source. These methods (Afraimovich et al., 2005, 2006) were applied for investigating the ionospheric response to large earthquakes. It was found that ionospheric disturbances have a form of a spherical wave propagating with a velocity of approximately $1000 \mathrm{~m} / \mathrm{s}$ from the "secondary" source localized over the epicenter on the ionospheric heights.

Optimum algorithms (Afraimovich et al., 2001c, 2002, $2005,2006)$ realized the coherent summation of the TEC series accounting for spatial-temporal parameters of disturbance. In order to determine the parameters of ionospheric perturbation the parameter values were varied. For each combination of the estimated parameter values, the maximum amplitude of cross-correlation integral for the coherent sum of all TEC series and the reference signal were calculated. The largest maximum value of cross-correlation integral corresponded to the best-fit perturbation parameters. For realizing the optimum algorithms it was considered, that the form of the ionospheric disturbance was known, represents a characteristic $N$-shape SAW and remains constant within the limits of the localization area. It is allowed to simulate a "copy" of the "signal" generated by the source and to use the "copy" as a reference signal in optimum algorithms for estimation the disturbance parameters.

However, in many cases the form of the initial signal generated by a source of disturbance is known only approximately (on the basis of early research of similar perturbations). In addition, the ionospheric perturbation itself represents a dispersive signal and leads to changes in the form of the ionospheric perturbation observed by separate elements of a GPS array. In that case the simulating the reference signal is impossible.

The quasi-optimum algorithm proposed in this paper should provide a method to determine the parameters of ionospheric perturbation (propagation phase velocity, location, height and "switch-on" time of the perturbation source) recorded in the near-zone of earthquake epicenter without prior information about the perturbation form and the site and time of the main shock of earthquake. We used this algorithm for localizing the source of ionospheric disturbances caused by the strong earthquake in the vicinity of Hokkaido Island which occurred on 25 September 2003, and the earthquake near the south coast of Honshu Island on September 5, 2004.

\section{The Definition of a Problem of Spatiotemporal Processing of TEC Measurements}

A majority of the models on the generation of ionospheric perturbations caused by an earthquake are derived 
from two main hypotheses. According to the first hypothesis the source located in the epicenter of an earthquake is the cause of coseismic ionospheric perturbations. The second hypothesis supposes that the coseismic ionospheric perturbations are generated by the secondary point source which is located at the height of maximum ionization of the ionosphere above the epicenter of an earthquake. Therefore, in this paper we have considered a general case when the cause of seismic ionospheric perturbation is the point source located at a certain height. It is necessary to determine the height of the point source of the ionospheric perturbation along with other unknown parameters (such as coordinates and switch-on time of the source and perturbation propagation velocity). This will provide not only the localization of the source of ionospheric perturbation but also the identification of the mechanism of the generation of ionospheric disturbance during an earthquake.

The measurements were carried out in the time interval from $t_{1}$ to $t_{2}$ along $M$ LOS formed by the GPS satellites and two-frequency multichannel GPS receivers in the region which includes a point source of ionospheric disturbance. The propagated ionospheric perturbation caused by a earthquake are manifested in the GPS phase measurements as additional phase delays of the navigation signal. On the basis of the algorithms (Calais and Minster, 1995; Afraimovich et al., 2001a) we calculated the series $I_{i}(t)$ of vertical values of TEC and the corresponding series of azimuth $\alpha_{i}$ and elevation angle $\theta_{i}$ for each LOS, where $i=1,2, \ldots, M-$ is the LOS number. Here we have cited the final formula of TEC value measuring:

$$
I=\frac{1}{40.308} \frac{f_{1}^{2} f_{2}^{2}}{f_{1}^{2}-f_{2}^{2}}\left[\left(L_{1} \lambda_{1}-L_{2} \lambda_{2}\right)+\text { const }+n L\right]
$$

where $L_{1} \lambda_{1}$ and $L_{2} \lambda_{2}$ are additional paths of the radio signal caused by the phase delay in the ionosphere, $(\mathrm{m}) ; L_{1}$ and $L_{2}$ represent the number of phase rotations at the frequencies $f_{1}$ and $f_{2} ; \lambda_{1}$ and $\lambda_{2}$ stand for the corresponding wavelengths, (m); const is unknown initial phase path; $n L$ are errors in determining the phase path. TEC $I$ is measured in $\mathrm{m}^{-2}$; const 40.308 has the dimension $\left[\mathrm{m}^{3} / \mathrm{s}^{2}\right]$.

For the elimination of spatial uncertainty at the localization of the observed ionospheric disturbance caused by the integrated character of TEC measurements, we considered that the TEC value was formed in one ionospheric point (IP) - a point of intersection of LOS with a plane on the height of a maximum ionization of ionospheric $F$-layer (as this area makes the basic contribution to the TEC formation) (Fig. 1). Using known geographical coordinates of GPS receivers, we transformed the series of azimuth $\alpha_{i}$ and elevation angle $\theta_{i}$ for the each LOS to the series $x_{i}(t), y_{i}(t)$, $z_{i}(t)$ of geocentric coordinates of corresponding IP. Then, for $M$ LOS we have obtained the GPS array consisting of $M$ elements.

Each TEC series $I_{i}(t)$ are realizations of a random process and represent the additive combination of the following components:

$$
I_{i}(t)=I_{T, i}(t)+\Delta I_{S, i}\left(t, V, \Phi_{e}, \Lambda_{e}, H_{e}\right)+\Delta I_{n, i}(t)
$$

where $I_{T, i}(t)$ represents the low-frequency TEC variations (trend) caused by regular changes (daily and seasonal)

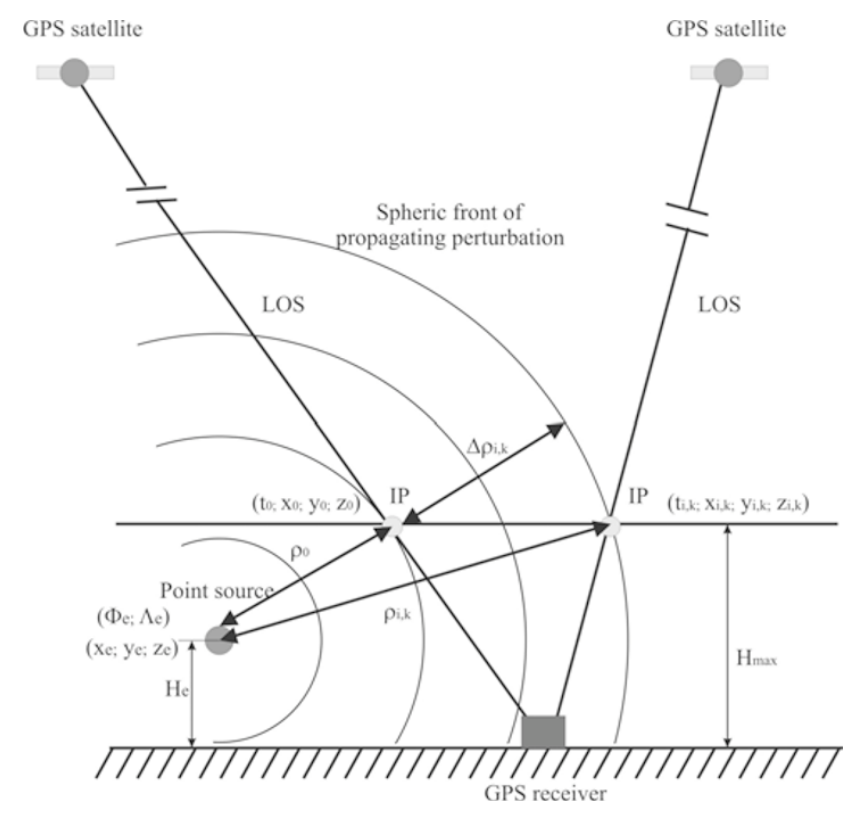

Fig. 1. The illustration of the model of spheric perturbation propagating from a point source in all directions with constant radial velocity. The geometry of GPS TEC measurements in vertical plane is shown. $t_{0}$ and $t_{i, k}$ are the moments of the perturbation arrival to the central and to the $i$-th ionospheric points (IP). $\Delta \rho_{i, k}$ is a difference of the distances $\rho_{0}$ and $\rho_{i, k}$ passed by the front of the model perturbation from the point source up to the central and up to the $i$-th elements of GPS array. $\left(x_{e}, y_{e}, z_{e}\right)$ and $\left(\Phi_{e}, \Lambda_{e}, H_{e}\right)$ are the geocentric and geographical coordinates of the source of model disturbance, respectively. $\left(x_{0}, y_{0}, z_{0}\right)$ and $\left(x_{i, k}, y_{i, k}\right.$, $\left.z_{i, k}\right)$ are the coordinates of the central and the $i$-th ionospheric points.

of electronic concentration; $\Delta I_{S, i}\left(t, V, \Phi_{e}, \Lambda_{e}, H_{e}\right)$ is the characteristic TEC perturbation ("useful signal") caused by earthquake; $V$ is the radial velocity of ionospheric perturbation propagating from the point source (i.e., a velocity along the straight line between the point source and IP of the GPS array); $\Phi_{e}, \Lambda_{e}, H_{e}$ are geographical coordinates and the height of the source of ionospheric disturbance; $\Delta I_{n, i}(t)$ is the noise high-frequency TEC fluctuations due to various sort of small-scale variations of electronic concentration.

As the duration of the ionospheric perturbation due to earthquake is known a priori, we filtered the TEC series $I_{i}(t)$ by removing the trend with a corresponding time window for selecting the characteristic ionospheric disturbance:

$$
\Delta I_{i}(t)=\Delta I_{S, i}\left(t, V, \Phi_{e}, \Lambda_{e}, H_{e}\right)+\Delta I_{n, i}(t)
$$

On the basis of the analysis of these series of TEC increments and corresponding series of coordinates $x_{i}(t)$, $y_{i}(t), z_{i}(t)$, it is necessary to give an estimation of the spatial-temporal characteristics of the ionospheric perturbation caused by earthquake. Namely, it is necessary to determine the perturbation propagation velocity, coordinates, height, and switch-on time of the source of ionospheric disturbance.

Further, we shall operate with a series of TEC increments, for the sake of convenience, we shall name the series $\Delta I(t)$ as TEC series. 


\section{The Auasi-Optimum Algorithm of Spatiotem- poral Processing of TEC Measurements}

This algorithm includes two stages: the experimental stage and the modeling stage. The experimental stage realizes coherent summation of all TEC series of the GPS array in order to obtain the accumulated TEC series for which the energy of total signal of the ionospheric response is maximum. For realization of the modeling stage we used a simplified mathematical model of the spheric perturbation propagating in all directions with constant radial velocity from a point source. The initial parameters of the model are the coordinates and height of the source and also the perturbation propagation velocity.

During the processing we varied values of the initial parameters of the model. For each combination we calculated times of arrival of the model perturbation to each ionospheric point of the GPS-array. We then determined the respective delays for the TEC series to be shifted in the summation and to obtain the modeled accumulated TEC series.

For each combination we computed the energy of the modeled accumulated TEC series. We considered that only certain combinations of the initial model parameters will reflect the spatial-temporal evolution of the experimentally observed perturbation in a optimal fashion. In this case the energy of the modeled accumulated TEC series will be close to the energy value of the accumulated series obtained on the experimental stage of processing. It was a criterion of conformity of model perturbation and the experimentally observed disturbance. We considered the respective values of the initial parameters of the model to be estimations of the spatio-temporal parameters of the experimentally observed perturbation.

\subsection{Experimental stage of processing}

We supposed that the high-frequency TEC fluctuations $\Delta I_{n, i}(t)$ are distributed according to the Gauss law with a zero expected value.

To estimate parameters of "useful signal", received on the background of gauss noise with spectral density $N_{n}$, it is enough to analyze the cross-correlation integral of the received realization of a signal and noise and the reference signal. For determining the spatial-temporal characteristics of the ionospheric perturbation observed by GPS array, this algorithm is reduced to a calculation of the cross-correlation integral $q$ of the coherent sum for all TEC series of GPS array and a reference signal $R_{0}(t)$ (under condition of a constancy of spectral density of noise $N_{n}$ in all TEC series) (Afraimovich et al., 2001c):

$$
q=\frac{1}{N_{n}} \int_{t_{1}}^{t_{2}} \sum_{i=1}^{M}\left[\Delta I_{i}(t) B_{i}\left(\tau_{i}\right)\right] R_{0}(t) d t
$$

where $B_{i}\left(\tau_{i}\right)$ is the multiplier of TEC series phasing.

Generally, for the nonharmonic signal, the multiplier $B_{i}\left(\tau_{i}\right)$ provides a shift in time of the $i$-th series $\Delta I_{i}(t)$ by $\tau_{i}$.

This algorithm is optimum under conditions that the "useful signal" is fully known (except for the parameters for which it is necessary to estimate), i.e., there is the exact copy $R_{0}(t)$ of the signal generated by the source of ionospheric disturbance.
However, in many cases, the form of the initial signal generated by a source of disturbance is known only approximately and, consequently, simulating the reference signal is impossible. It will then be more expedient to use the quasi-optimum algorithm which does not require prior information about the perturbation form.

For realization of the quasi-optimum algorithm we have written the expression (4) in the form of:

$$
\begin{gathered}
q=\sum_{i=1}^{M} q_{i} \\
q_{i}=\frac{1}{N_{n}} \int_{t_{1}}^{t_{2}} \Delta I_{\Sigma, i}\left(t, \tau_{i}\right) R_{0}(t) d t
\end{gathered}
$$

where $q_{i}$ is a cross-correlation integral on the $i$-th iterations of summation, and $\Delta I_{\Sigma, i}$ - is stacked series on the $i$-th iterations of summation.

At the unknown form of an initial signal we used the stacked series as $R_{0}(t)$ in expression (6) for $q_{i}$ and have written down the cross-correlation integral in the form of:

$$
q_{i}=\frac{1}{N_{n}} \int_{t_{1}}^{t_{2}}\left[\Delta I_{\Sigma, i}\left(t, \tau_{i}\right)\right]^{2} d t
$$

In that case, expression (7) for $q_{i}$ represents the relation of energy of the stacked TEC series on the $i$-th iteration of summation to spectral density of noise. We determined the evaluation value $\hat{\tau}_{i}$ of the time delay on which it is necessary to shift the $i$-th TEC series at the summation from the maximum of $q_{i}$ value:

$$
\frac{d q_{i}}{d \tau_{i} \hat{\tau}_{i}=\tau_{i}}=0
$$

For the determination of conditions of the $q_{i}$ maximization we have written the expression for the stacked series on the $i$-th iteration of summation as:

$$
\Delta I_{\Sigma, i}(t)=\Delta I_{\Sigma, i-1}(t)+\Delta I_{i}\left(t-\tau_{i}\right)
$$

where $\Delta I_{\Sigma, i-1}$ is the stacked series on the $(i-1)$-th iteration of summation.

Taking into account the expression (9) and (7), we can write for $q_{i}$ :

$$
\begin{aligned}
q_{i}= & \frac{1}{N_{n}}\left[\int_{t_{1}}^{t_{2}} \Delta I_{\Sigma, i-1}^{2}(t) d t+2 \int_{t_{1}}^{t_{2}} \Delta I_{\Sigma, i-1}(t) \Delta I_{i}(t) d t\right. \\
& \left.+\int_{t_{1}}^{t_{2}} \Delta I_{i}^{2}(t) d t\right]
\end{aligned}
$$

In expression (10) the first summand represents full energy of the stacked series on the $(i-1)$-th iteration of summation. The third summand of expression (10) represents energy of the $i$-th TEC series. These quantities do not depend on the value of delay $\tau_{i}$. The second summand represents the cross-correlation function of the stacked series on the $(i-1)$-th iteration of summation and the $i$-th TEC series:

$$
r_{i}=\int_{t_{1}}^{t_{2}} \Delta I_{\Sigma, i-1}(t) \Delta I_{i}(t) d t
$$



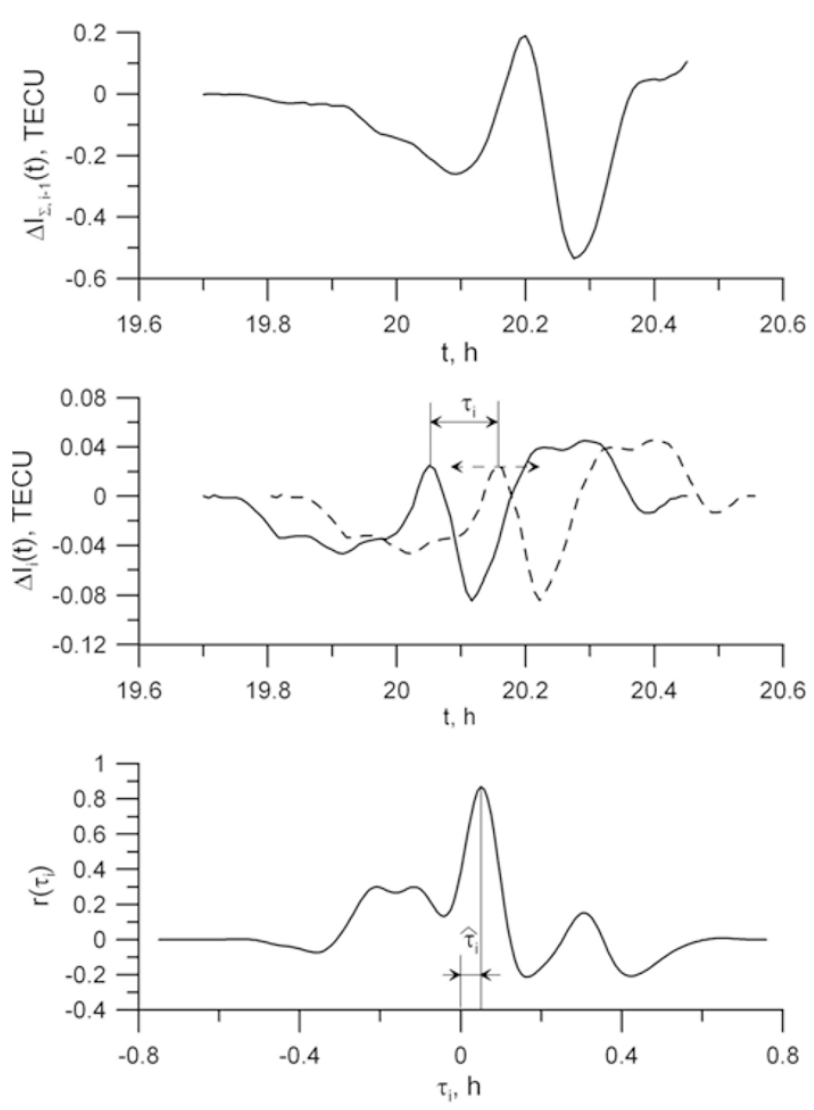

Fig. 2. The illustration of the concept of the time delay estimation of TEC series during the coherent accumulation of series at the experimental stage of processing. $\Delta I_{\Sigma, i-1}(t)$ is the stacked series on the $(i-1)$-th iteration of summation. The $i$-th TEC series is marked as $\Delta I_{i}(t), r\left(\tau_{i}\right)$ is the cross-correlation function of the $\Delta I_{\Sigma, i-1}(t)$ and $\Delta I_{i}(t)$ series. $\tau_{i}$ is a current value of delay of the $i$-th TEC series. The evaluation value of time delay of the $i$-th TEC series corresponding to the maximum of $r\left(\tau_{i}\right)$ is denoted by $\hat{\tau}_{i}$.

Then, for the $q_{i}$ maximization it is enough to maximize the second summand of expression (10):

$$
\frac{d r_{i}}{d \tau_{i} \hat{\tau}_{i}=\tau_{i}}=0
$$

For this purpose we searched all possible values of $\tau_{i}$ in limits $\pm\left(t_{2}-t_{1}\right)$. For each $\tau_{i}$ we determined the value of $r_{i}$.

We considered the $\hat{\tau}_{i}$ value for which the maximum of $r_{i}$ was reached to be the estimation of delay of $i$-th TEC series at summation. At the end, we shifted the $i$-th TEC series exactly by this delay to obtain the stacked series on the $i$-th iteration of summation:

$$
\Delta I_{\Sigma, i}(t)=\Delta I_{\Sigma, i-1}(t)+\Delta I_{i}\left(t-\hat{\tau}_{i}\right)
$$

The concept of the delay estimation is illustrated on the graphs shown in Fig. 2.

In general, the expression for the stacked series, obtained as a result of summation of $i$ TEC series may be written down in the form of:

$$
\Delta I_{\Sigma, i}(t)=\Delta I_{0}(t)+\sum_{j=1}^{i} \Delta I_{j}\left(t-\hat{\tau}_{j}\right)
$$

where $j=1,2, \ldots, i$ - is the TEC series number, $\Delta I_{0}(t)$ is some central series to which all other series are summed.
Accordingly, the expression for the accumulated series, obtained as a result of summation of all $M$ TEC series of the GPS array, may be written down in the form of:

$$
\Delta I_{\Sigma}(t)=\Delta I_{0}(t)+\sum_{i=1}^{M} \Delta I_{i}\left(t-\hat{\tau}_{i}\right)
$$

It is necessary to pay special attention to a choice of central series as the one chosen should reflect in the best way possible the unknown form of the ionospheric perturbation. Therefore, as the central series we selected the one the most similar to all other series $\Delta I_{i}(t)$. For this purpose, we calculated the average factor of correlation of each series with all other TEC series:

$$
K_{i}=\frac{1}{M} \sum_{l=1}^{M} k_{i, l}
$$

where $l=1,2, \ldots, M$ - is the TEC series number, $k_{i, l}$ is the maximal value of function of correlation of the $\Delta I_{i}(t)$ and the $\Delta I_{l}(t)$ series. The series for which the $K_{i}$ value was maximal we selected as the central one.

The value $q=q_{\max }$, obtained according to the algorithm (5)-(16), is the greatest possible value of $q$, which may be reached at accumulation of the TEC series $\Delta I_{i}(t)$. As a result we obtained the accumulated series $\Delta I_{\Sigma}(t)=$ $\Delta I_{S, \Sigma}(t)+\Delta I_{n}(t)$, which include the total "useful signal" $\Delta I_{S, \Sigma}(t)$ and the averaged noise fluctuations $\Delta I_{n}(t)$.

Only signal variations regularly repeating in all TEC series make the basic contribution to the formation of total "useful signal", $\Delta I_{S, \Sigma}$, at summation. Random outliers, changes of the signal form, and noise fluctuations of the separate series are canceled out each other. Only in this case will the task of maximization of energy of accumulated series be solved. The total "useful signal" represents an average shape of the ionospheric responses, observed by a separate LOS of GPS array.

We selected the moment $t_{0}$ of observation of maximal value of the total "useful signal" as a characteristic point of the complete stacked series $\Delta I_{\Sigma}$. At the summation, all TEC series were summed to the central one $\Delta I_{0}$; therefore, we considered the coordinates $\left(x_{0}, y_{0}, z_{0}\right)$ of the IP corresponding to the central series as the spatial position of perturbation, observed by the central element of GPS array at $t_{0}$ instant of time.

\subsection{The modeling stage}

We used the simplified mathematical model of the spheric perturbation propagating in all directions with constant radial velocity from a point source (Fig. 1). The initial parameters of model are the geographical coordinates $\Phi_{e}$, $\Lambda_{e}$, and height $H_{e}$ of the source and also the perturbation propagation velocity, $V$.

We found the evaluation values of the spatial-temporal parameters of the observed disturbance by a numerical method. For this purpose, within the assigned area of uncertainty with the assigned step we selected all possible values of the initial parameters of the model. For each combination we calculated times $t_{i, k}$ of arrival of the model perturbation to each $i$-th ionospheric point of the GPS-array. We chose the modeled times $t_{i, k}$ as the time instant of the $k$-th sample 
of the $i$-th TEC series $\Delta I_{i}$ reasoning from the minimization of expression:

$$
\left(t_{i, k}-t_{0}\right)-\frac{\Delta \rho_{i, k}}{V}=\min
$$

where $\Delta \rho_{i, k}=\rho_{i, k}-\rho_{0}$ is a difference of the distances passed by the front of the model perturbation from the point source up to central (at the instant $t_{0}$ ) and up to the $i$-th (at the instant $t_{i, k}$ ) elements of GPS array.

Then, the expressions for these distances can be written down as:

$$
\begin{aligned}
\rho_{i, k} & =\sqrt{\left(x_{i, k}-x_{e}\right)^{2}+\left(y_{i, k}-y_{e}\right)^{2}+\left(z_{i, k}-z_{e}\right)^{2}} \\
\rho_{0} & =\sqrt{\left(x_{0}-x_{e}\right)^{2}+\left(y_{0}-y_{e}\right)^{2}+\left(z_{0}-z_{e}\right)^{2}}
\end{aligned}
$$

where $\left(x_{e}, y_{e}, z_{e}\right)$ are the geocentric coordinates of the source of model disturbance which are obtained by the standard transformations of the current value of geographical coordinates $\left(\Phi_{e}, \Lambda_{e}, H_{e}\right)$ of model source; $\left(x_{i, k}, y_{i, k}, z_{i, k}\right)$ are the coordinates of the $i$-th element of the GPS array at the $t_{i, k}$ instant of time.

The presented algorithm (17)-(19) of a choice of the necessary sample of the TEC series is iterative. It excludes the necessity of the additional mathematical account of movement of GPS satellite as the algorithm operates with the already known coordinates and temporal parameters of the GPS array elements (IP trajectories). The choice of the necessary sample was carried out by testing each IP position at each time instant reasoning from a minimization of expression (17).

We determined the respective modeled delays $\tau_{i}^{m}$ on which it was necessary to shift the $i$-th TEC series at summation for obtaining the accumulated TEC series. We determined the modeled time delays as the difference in time between the instant $t_{i, k}$ and the instant $t_{0}$ of observation of the perturbation by the central element of the GPS-array:

$$
\tau_{i}^{m}=t_{i, k}-t_{0}
$$

Taking into account the obtained modeled delays $\tau_{i}^{m}$ of the TEC series, we formed the modeled accumulated series by analogy to (15):

$$
\Delta I_{\Sigma}^{m}(t)=\Delta I_{0}(t)+\sum_{i=1}^{M} \Delta I_{i}\left(t-\tau_{i}^{m}\right)
$$

For each combination of the initial parameters we computed the energy of the modeled accumulated TEC series:

$$
\begin{aligned}
& q^{m}\left(V, \Phi_{e}, \Lambda_{e}, H_{e}\right) \\
& =\frac{1}{N_{n}} \sum_{i=1}^{M} \int_{t_{1}}^{t_{2}}\left[\Delta I_{\Sigma, i}^{m}\left(t, \tau_{i}^{m}\left(V, \Phi_{e}, \Lambda_{e}, H_{e}\right)\right)\right]^{2} d t
\end{aligned}
$$

where

$$
\Delta I_{\Sigma, i}^{m}(t)=\Delta I_{0}(t)+\sum_{j=1}^{i} \Delta I_{j}\left(t-\tau_{j}^{m}\left(V, \Phi_{e}, \Lambda_{e}, H_{e}\right)\right)
$$

represents the modeled stacked series on the $i$-th iteration of summation.
We considered that only for a certain combination of values of the initial parameters will the dynamics of model perturbation reflect the spatial-temporal evolution of the experimentally observed perturbation in an optimal fashion. At that combination, the energy $q^{m}$ of the modeled accumulated TEC series will close to energy value $q_{\max }$ of the accumulated series obtained on the experimental stage of processing.

By analogy to the method COPHASE (Montes and Posmentier, 1971) developed for the analysis of seismic wave packages, we used the relation of energy values of the calculated and experimental stacked series as a criterion of conformity of model perturbation and the experimentally observed disturbance:

$$
\begin{aligned}
& C\left(V, \Phi_{e}, \Lambda_{e}, H_{e}\right) \\
& =\frac{q^{m}\left(V, \Phi_{e}, \Lambda_{e}, H_{e}\right)}{q_{\max }} \\
& =\frac{\sum_{i=1}^{M} \int_{t_{1}}^{t_{2}}\left[\Delta I_{\Sigma, i}^{m}\left(t, \tau_{i}^{m}\left(V, \Phi_{e}, \Lambda_{e}, H_{e}\right)\right)\right]^{2} d t}{\sum_{i=1}^{M} \int_{t_{1}}^{t_{2}}\left[\Delta I_{\Sigma, i}\left(t, \hat{\tau}_{i}\right)\right]^{2} d t}
\end{aligned}
$$

The respective values of the initial parameters of model, for which the maximum of criterion $C$ was reached, were considered to be estimations $\hat{V}, \hat{\Phi}_{e}, \hat{\Lambda}_{e}, \hat{H}_{e}$ of the spatiotemporal parameters of the experimentally observed ionospheric perturbation.

The criterion $C$ represents the normalized multivariate function of uncertainty changing in limits from 0 up to 1 . Application of such a criterion excludes the necessity of measurement of spectral density of noise fluctuations $N_{n}$ as this quantity is canceled out at a calculating of the relation C.

We also determined the "switch-on" time $t_{e}$ of a source of the ionospheric perturbation within the framework of the accepted mathematical model on the basis of the obtained estimations of perturbation source coordinates and height, propagation velocity of the perturbation and also the known coordinates $\left(x_{0}, y_{0}, z_{0}\right)$ and the instant $t_{0}$ of the disturbance observation by the central element of a GPS-array:

$$
t_{e}=t_{0}-\frac{\sqrt{\left(x_{0}-\hat{x}_{e}\right)^{2}+\left(y_{0}-\hat{y}_{e}\right)^{2}+\left(z_{0}-\hat{z}_{e}\right)^{2}}}{\hat{V}}
$$

where $\left(\hat{x}_{e}, \hat{y}_{e}, \hat{z}_{e}\right)$ are evaluation values of geocentric coordinates of the source of ionospheric perturbation which are obtained by standard transformations of estimations $\left(\hat{\Phi}_{e}\right.$, $\left.\hat{\Lambda}_{e}, \hat{H}_{e}\right)$.

\section{Experimental Check of the Algorithm}

For experimental checking of the proposed algorithm we processed the ionospheric GPS-measurements carried out by Japanese stations of IGS network during two strong earthquakes: the earthquake in the vicinity of Hokkaido Island on September 25, 2003 (Hokkaido earthquake) and the earthquake near the south coast of Honshu Island on September 5, 2004 (Honshu earthquake).

The experiment geometry during the Hokkaido earthquake is presented in Fig. 3. The earthquake epicenter $\left(41.8^{\circ} \mathrm{N}, 143.85^{\circ} \mathrm{E}\right)$ was at the depth of $27-33 \mathrm{~km}$ underwater between the Kuril and Japanese Trenches (it is shown in 


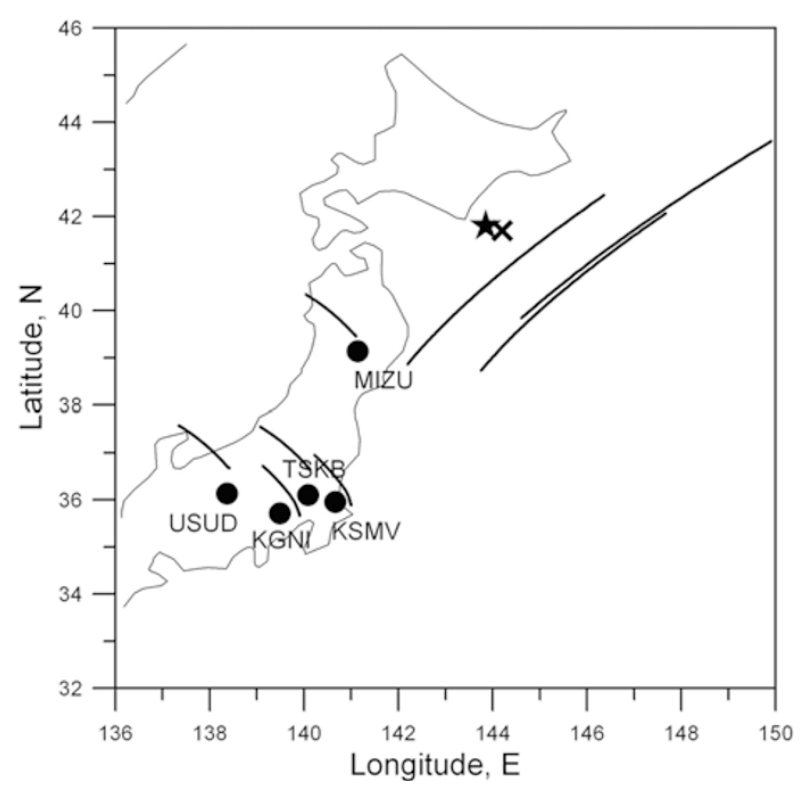

Fig. 3. Experimental geometry of GPS measurements during the Hokkaido earthquake on September 25, 2003. GPS stations positions are marked by dots, the names of the sites are written nearby. An asterisk shows the epicenter's location. The movement of trajectories of the subionospheric points are shown by continuous thick lines. The calculated location of the source of ionospheric perturbation is marked by a thick dagger.

the figure by an asterisk). Ten fore-shocks with magnitude $M=3.9-5.7$ were registered in the area of the earthquake epicenter. The earthquake was accompanied by tsunami along all coast of the Japanese islands. In the 5 days following the main earthquake, more than 100 after-shocks with a magnitude $M=3.8-7.4$ were registered (http://www.neic. cr.usgs.gov).

In the second experiment, the earthquake took place near the south coast of Japanese Island Honshu (33.19N, $137.06 \mathrm{E})$ at the depth $10 \mathrm{~km}$ underwater. The main shock of the earthquake with magnitude $M=7.4$ took place at 14:57:18 (14.955) UT. Figure 4 shows the corresponding geometry of this experiment. By analogy to Fig. 3, in Fig. 4, an asterisk shows the earthquake epicenter position.

In both cases the level of geomagnetic disturbances was quite moderate: during the Hokkaido earthquake the $D_{s t}$ variations were within $-21 / 11 \mathrm{nT}$ and the $K_{p}$ index varied from 1 to 4; during the Honshu earthquake the corresponding parameters were $D_{s t}=-38 / 4 \mathrm{nT}$ and $K_{p}=1-$ 3. The data on the geomagnetic indices $D_{s t}$ and $K_{p}$ were taken from the WDC site (http://www.wdc.rl.ac.uk/cgibin/wdccl/secure/wdcdata).

The data of GPS-measurements used in our research are presented for free use on a site (http://sopac.ucsd.edu/cgibin/dbDataByDate.cgi) in standard format RINEX.

In our experiments, the measurements were carried out by GPS-stations USUD, MIZU, TSKB, KGNI, KSMV. Dots on Figs. 3 and 4 show the GPS stations position. The movements of trajectories of the projections of the IP on the Earth's surface (subionospheric points) are shown in Figs. 3 and 4 by continuous thick lines.

For the Hokkaido earthquake shown in Fig. 5, four series of TEC increments reconstructed from GPS-measurements

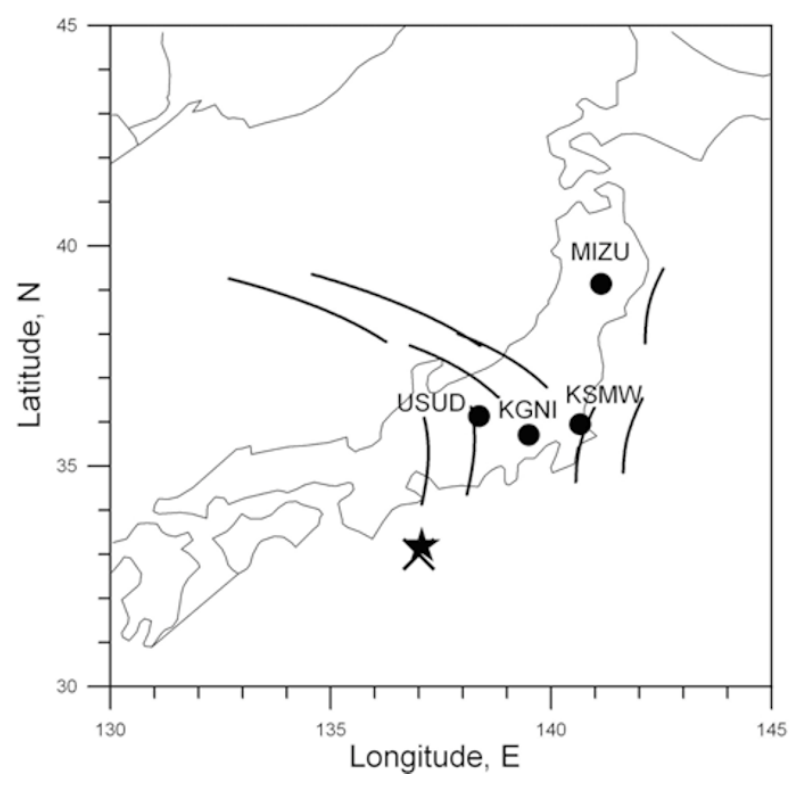

Fig. 4. Geometry of GPS measurements during the Honshu earthquake on September 5, 2004. The designations are same as in Fig. 3.

along the LOS KGNI - PRN13 (panel a), KGNI - PRN24 (panel b), TSKB - PRN13 (panel c), KSMV - PRN24 (panel d) are presented. One can see that the amplitude of TEC responses to the earthquake does not exceed 0.1 TECU, and the value of perturbation period lies within the range of 1015 minutes. The form of ionospheric responses observed along the separate LOS is variable but, nevertheless, it is possible to select a regular TEC variations repeating in each ionospheric response.

The analysis of average correlation factors of everyone series $\Delta I(t)$ with all other series has shown that the series $\Delta I_{0}(t)$ obtained along the LOS KSMV - PRN24 (Fig. 5(d)) is the most similar to all other TEC series. For this series the value of the average factor of correlation is the largest and amounts to $K=0.828$. Therefore, during the spatiotemporal processing we used the LOS KSMV - PRN24 as a central element and the corresponding TEC series as a central series of the GPS array.

The continuous line in Fig. 5(e) shows the accumulated series $\Delta I_{\Sigma}(t)$, obtained on the experimental stage of processing according to algorithm (5)-(16). The graph of the modeled accumulated series $\Delta I_{\Sigma}^{m}(t)$ for which the maximum of criterion parameter $C$ was reached is marked on Fig. 5(e) by a dash line. The values of initial parameters of model at which the maximum $C_{\max }=0.9804$ of criterion $C$ was reached were considered to be the estimations of spatio-temporal parameters $\hat{V}, \hat{\Phi}_{e}, \hat{\Lambda}_{e}, \hat{H}_{e}$ of perturbation experimentally observed during the Hokkaido earthquake. At that the correlation factor of the series $\Delta I_{\Sigma}(t)$ with the series $\Delta I_{\Sigma}^{m}(t)$ make up $k_{\Sigma}=0.9845$.

By analogy to Fig. 5 the TEC measurements carried out along the LOS USUD - PRN07 (panel a), KSMV -PRN07 (panel b), MIZU - PRN07 (panel c), KSMV - PRN31 (panel d) during the Honshu earthquake are presented in Fig. 6. In Fig. 6 one can see that the amplitude of the TEC responses to the Honshu earthquake is less of a corresponding parameter of Hokkaido earthquake responses and does not exceed 

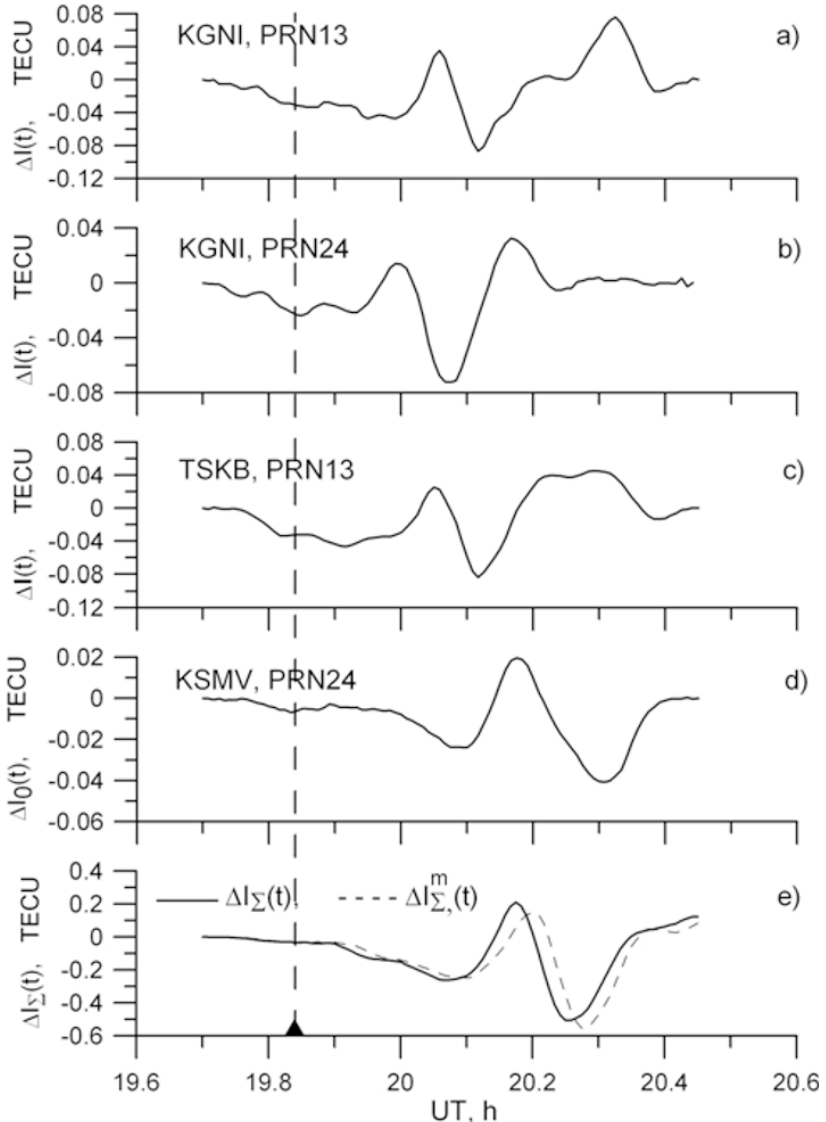

Fig. 5. The series of TEC increments $\Delta I(t)$ reconstructed from GPS-measurements along the LOS KGNI-PRN13 (a), KGNI-PRN24 (b), TSKB-PRN13 (c), KSMV-PRN24 (d) for the Hokkaido earthquake. The series $\Delta I_{0}(t)$ for LOS KSMV-PRN24 is a central series of GPS array. On the panel e the continuous line shows the accumulated TEC series $\Delta I_{\Sigma}(t)$, obtained on the experimental stage of processing. The dashed line on the panel (e) marks the modeled accumulated TEC series $\Delta I_{\Sigma}^{m}(t)$ for which the maximum of criterion parameter $C$ was reached. The moment of the main shock of Hokkaido earthquake is marked by shaded triangle.

0.04 TECU. The ionospheric responses observed along the separate LOS are very different in form. However, we used the series KSMV - PRN31 as the central series of GPSarray during the processing. The value of the average correlation factors of this series with all other series make up $K=0.6577$. In this experiment the maximum of criterion parameter $C$ has reached value $C_{\max }=0.8371$. The corresponding accumulated series $\Delta I_{\Sigma}(t)$ and the modeled accumulated series $\Delta I_{\Sigma}^{m}(t)$ are marked in Fig. 6(e) by a continuous line and dashed line respectively. The correlation factor of these series make up $k_{\Sigma}=0.9523$.

It is necessary to note that, in both cases, the total "useful signal" in amplitude is 5- to 20-fold higher than the ionospheric responses to the earthquake observed along the separate LOS.

One can see from Fig. 5(e) that the shape of a total "useful signal", which represents an average shape of separate ionospheric responses, obtained for Hokkaido earthquake is close to the characteristic $N$-shape wave. For the Honshu earthquake, the shape of a total "useful signal" is more complex (Fig. 6(e)). This is due to the fact that the shape of
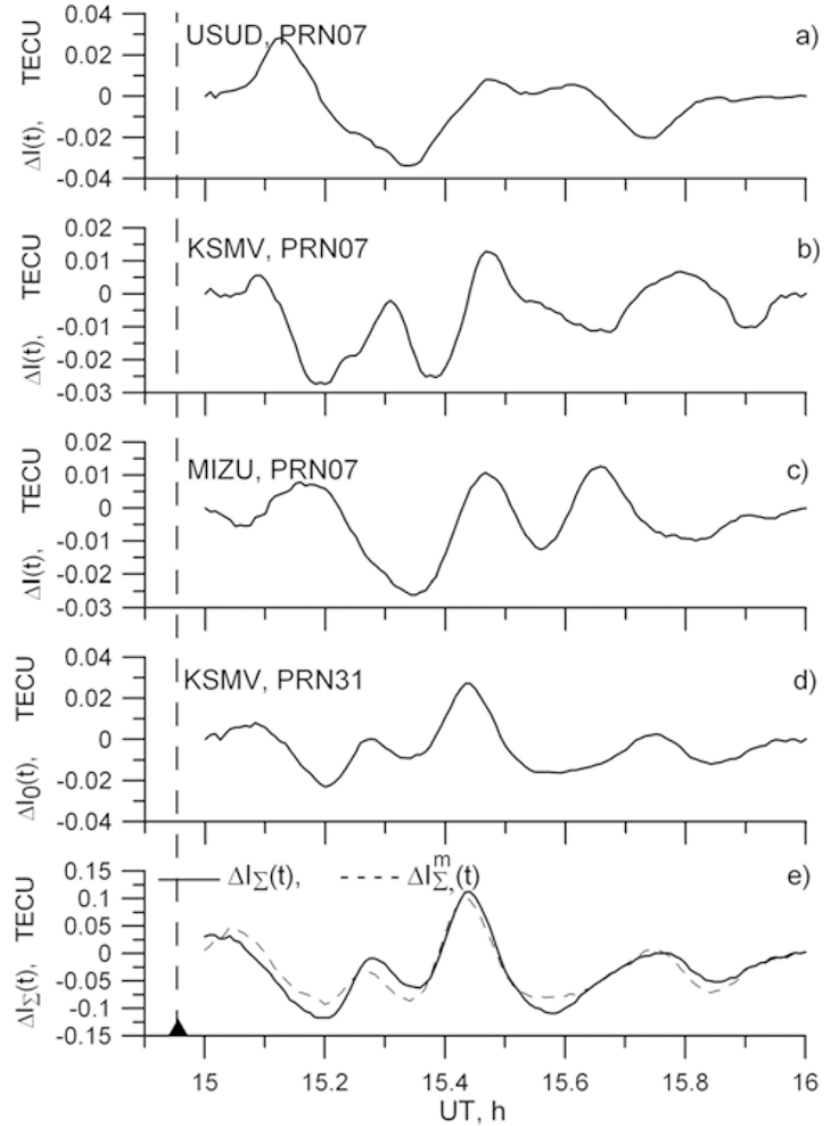

Fig. 6. The series of TEC increments $\Delta I(t)$ reconstructed from GPS-measurements along the LOS USUD-PRN07 (a), KSMV-PRN07 (b), MIZU-PRN07 (c), KSMV-PRN31 (d) during the Honshu earthquake. The series $\Delta I_{0}(t)$ for LOS KSMV-PRN31 is a central series of GPS array. On the panel e the designations of the accumulated TEC series $\Delta I_{\Sigma}(t)$ and the modeled accumulated TEC series $\Delta I_{\Sigma}^{m}(t)$ are same as in Fig. 5.

the ionospheric perturbation is distorted strongly during its propagation. The form of ionospheric responses, observed along the separate LOS, illustrate this fact in Fig. 6.

The analysis of graphs from Fig. 5(e) and the results of $C_{\max }$ and $k_{\Sigma}$ allow us to draw the conclusion that these is a degree of conformity between the ionospheric disturbance, experimentally observed during the Hokkaido earthquake, and the model of the spherical perturbation propagating with constant velocity $\hat{V}$ from a point source with coordinates $\hat{\Phi}_{e}, \hat{\Lambda}_{e}, \hat{H}_{e}$ is very high.

The obtained evaluation values of coordinates of the source of the ionospheric disturbance $\hat{\Phi}_{e}=41.7^{\circ} \pm 0.1^{\circ} \mathrm{N}$ and $\hat{\Lambda}_{e}=144.2^{\circ} \pm 0.1^{\circ} \mathrm{E}$ (the source is marked in Fig. 3 by a bold dagger) are in agreement with the position of the Hokkaido earthquake epicenter $\left(41.8^{\circ} \mathrm{N}, 143.85^{\circ} \mathrm{E}\right)$. The accuracy of the determination of the source coordinates is $33 \mathrm{~km}$.

The estimation of the height of the perturbation source $(340 \pm 80 \mathrm{~km})$ confirms a conception of generation of the coseismic ionospheric perturbations from a "secondary" source localized over the epicenter at the ionospheric heights (Francis, 1975; Hocke and Schlegel, 1996; Afraimovich et al., 2001b; Rudenko and Uralov, 1995) and is in agreement with results (Afraimovich et al., 2005, 


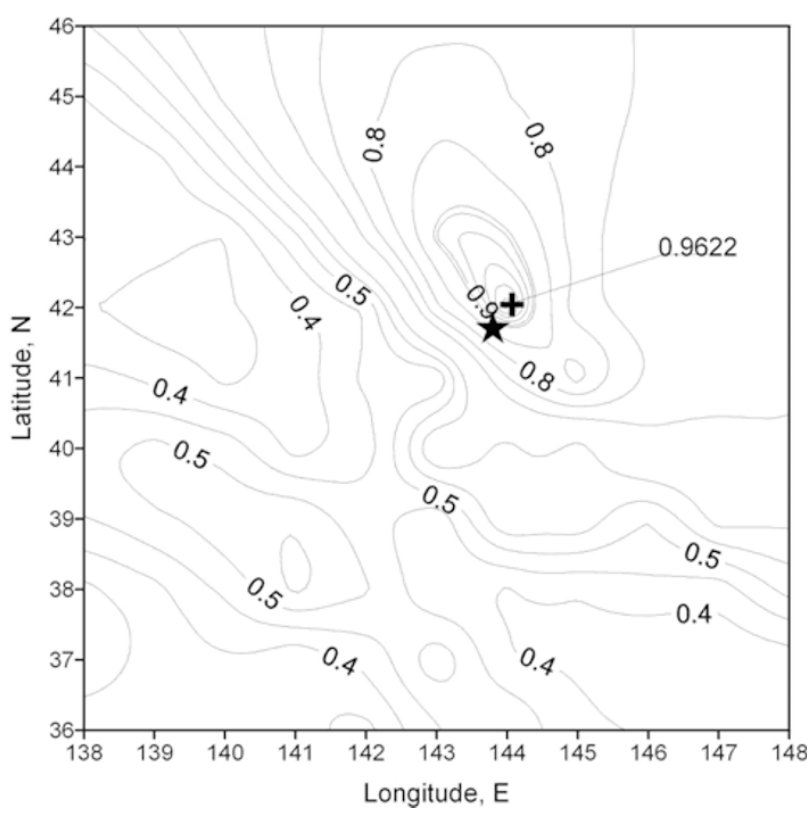

Fig. 7. The criterion parameter dependence on the geographic coordinates of the source of the ionospheric perturbation $C\left(\Phi_{e}, \Lambda_{e}\right)$ for the Hokkaido earthquake. The graph is constructed at a primary uncertainty of the search of the source coordinates $\left(36^{\circ}-46^{\circ} \mathrm{N}\right.$ and $\left.138^{\circ}-148^{\circ} \mathrm{E}\right)$ and the value of the search step $1^{\circ}$. A thick dagger shows the point $42^{\circ} \mathrm{N}$, $144^{\circ} \mathrm{E}$ in vicinity of which the maximal values of parameter $C$ are concentrated. An asterisk shows the epicenter's location.

2006) obtained earlier using the optimum algorithm. This theory is also verified by the fact that the delay between "switch-on" time of the "secondary" source (19.9312 UT) and the time of main shock of the earthquake (19.8352 UT) is $346 \mathrm{~s}$.

The obtained evaluation value of perturbation propagation velocity $\hat{V}=820 \pm 60 \mathrm{~m} / \mathrm{s}$ is close to the sound speed at the ionospheric heights that confirms the mechanism of transformation of initial energy of the main shock of earthquake by means of acoustic waves.

For the Honshu earthquake the accuracy of determination of coordinates of ionospheric perturbation source $\left(33.0^{\circ} \pm\right.$ $0.1^{\circ} \mathrm{N}, 137.0^{\circ} \pm 0.1^{\circ} \mathrm{E}$ ) is not worse than the corresponding accuracy of the Hokkaido experiment and is $27 \mathrm{~km}$ relative to earthquake epicenter. The obtained value of the height of the perturbation source is $370 \pm 130 \mathrm{~km}$. The delay between "switch-on" time of the source of ionospheric perturbation (15.0959 UT) and the time of main shock of the earthquake (14.955 UT) is $507 \mathrm{~s}$. It is also confirms a conception of generation of the coseismic ionospheric perturbations from a "secondary" source. The evaluation value of the perturbation propagation velocity $(460 \pm 40 \mathrm{~m} / \mathrm{s})$ is less than the result obtained in first experiment, but it is also within the range of sound speed at the ionospheric heights. The parameters $C_{\max }$ and $k_{\Sigma}$ show that the degree of conformity of the model of the spherical perturbation propagating with constant velocity from a point source and the ionospheric disturbance experimentally observed during the Honshu earthquake is lower than in the Hokkaido experiment.

In order to illustrate convergence of the process of the search and the solution uniqueness we show on Fig. 7 the graph $C\left(\Phi_{e}, \Lambda_{e}\right)$ (at $V=\hat{V}$ and $H_{e}=\hat{H}_{e}$ ) in the form

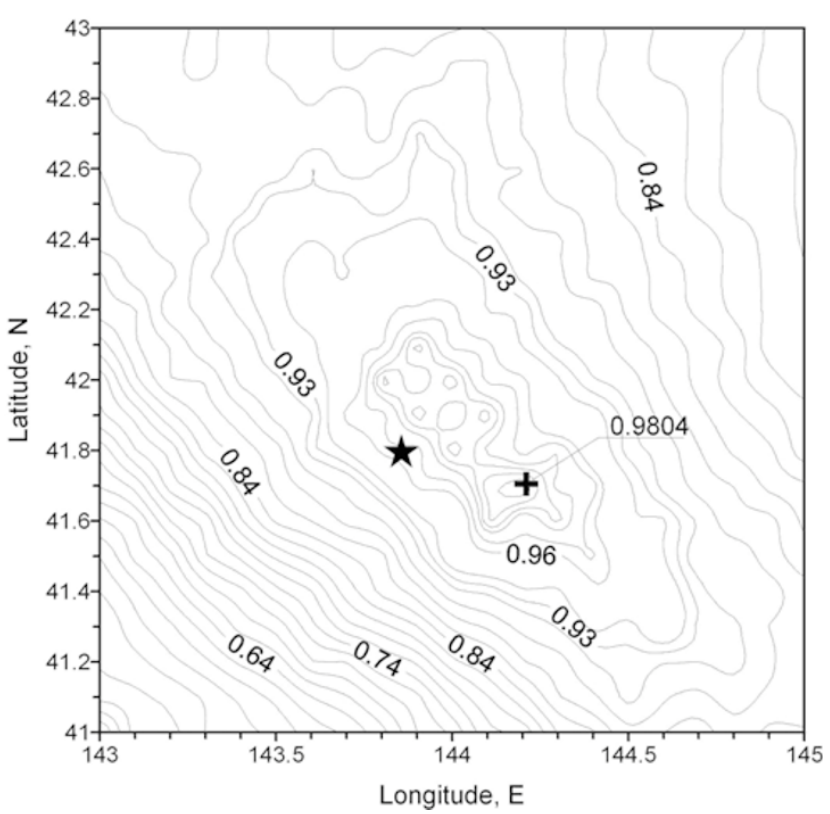

Fig. 8. The more exact graph of the criterion parameter dependence on the geographic coordinates of the source of ionospheric perturbation $C\left(\Phi_{e}, \Lambda_{e}\right)$ for the Hokkaido earthquake in about a point of maximal values of parameter $C$. The graph is constructed at smaller search uncertainty $\left(41^{\circ}-43^{\circ} \mathrm{N}\right.$ and $\left.143^{\circ}-145^{\circ} \mathrm{E}\right)$ and more exact search step $0.1^{\circ}$. The point $41.7^{\circ} \mathrm{N}, 144.2^{\circ} \mathrm{E}$ of the greatest value of criterion parameter is marked by a dagger. An asterisk shows the epicenter's location.

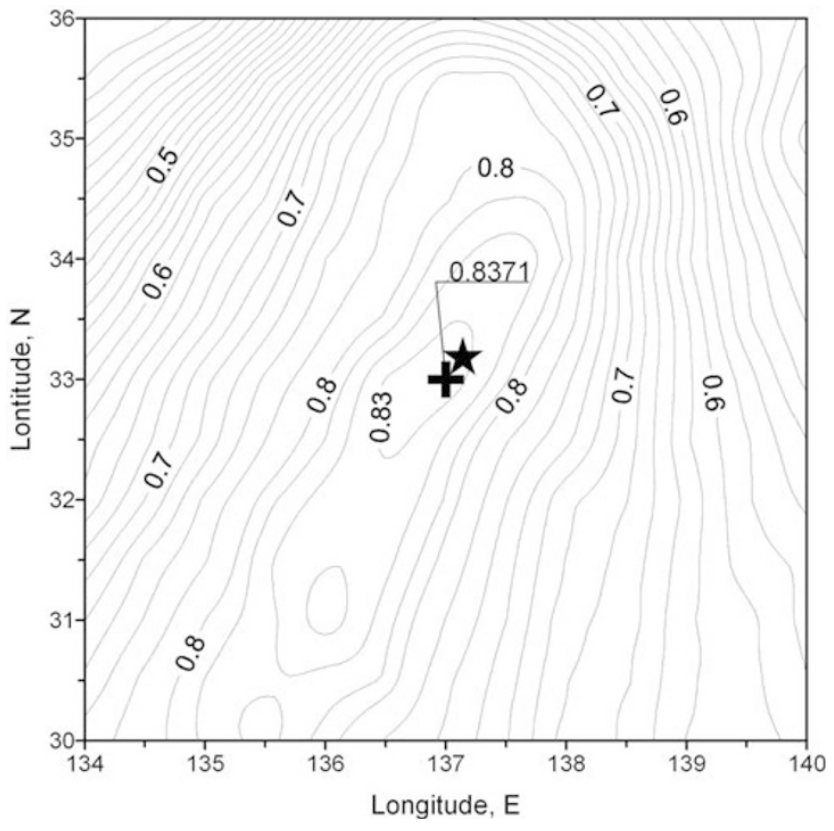

Fig. 9. Same as in Fig. 7, but for the Honshu earthquake. The graph is constructed at a primary uncertainty of the search of the source coordinates $\left(30^{\circ}-36^{\circ} \mathrm{N}\right.$ and $\left.134^{\circ}-140^{\circ} \mathrm{E}\right)$ and the value of the search step $0.1^{\circ}$. The maximal value of criterion parameter $C$ is reached at the point $33.0^{\circ} \mathrm{N}$, $137.0^{\circ} \mathrm{E}$.

of an equal level lines for Hokkaido earthquake. The graph was constructed at a primary uncertainty of the search of the coordinates of the source of perturbation $\left(36^{\circ}-46^{\circ} \mathrm{N}\right.$ and $\left.138^{\circ}-148^{\circ} \mathrm{E}\right)$ and the value of the search step $1^{\circ}$. One can see that the maximal values of $C\left(\Phi_{e}, \Lambda_{e}\right)$ are concentrated 


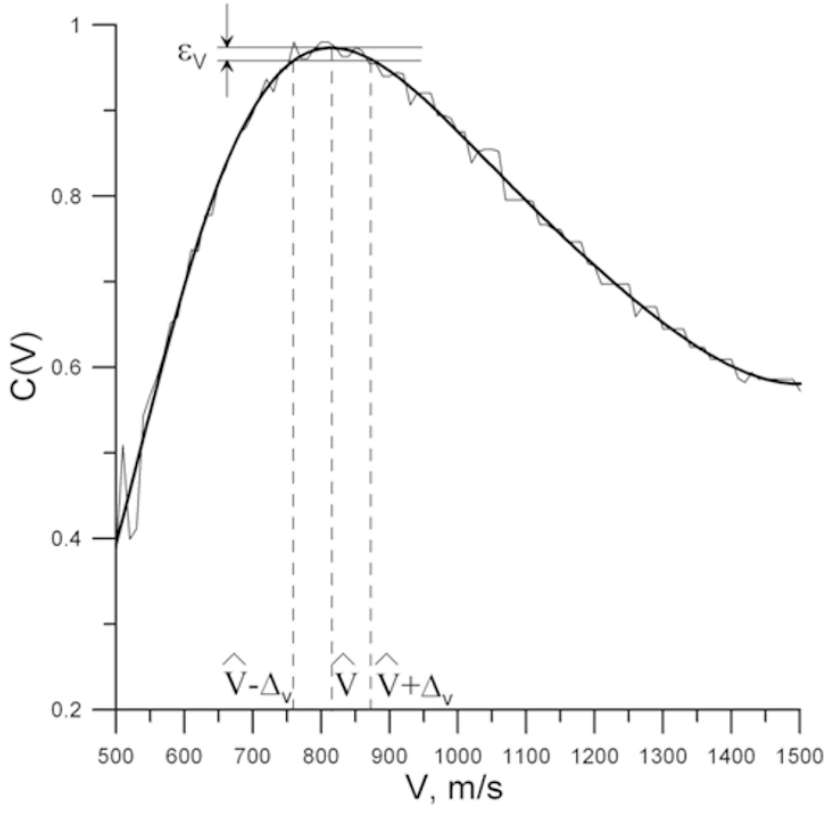

Fig. 10. The graph of the criterion parameter dependence on the propagation velocity of the ionospheric perturbation $C(V)$ for the Hokkaido earthquake. The low-frequency "signal" component $C_{s}(V)$ obtained by polynomial approximation of the initial function $C(V)$ is marked by a bold curve. The velocity value $\hat{V}=820 \mathrm{~m} / \mathrm{s}$ at which the maximum of the functions $C_{s}(V)$ was reached is an estimation of parameter $V$. A line parallel to the axis $V$ on the level $\epsilon_{V}=0.02$ below the maximum of the "signal" function is used to determine the confidential interval of the estimation. The $\epsilon_{V}$ is an amplitude of the "noise" component $C_{n}(V)$ of initial dependence $C(V)$. The bounds of confidential interval $\Delta_{V}= \pm 60 \mathrm{~m} / \mathrm{s}$ also evaluation value $\hat{V}$ are marked by a dashed lines.

in a vicinity of the point $42^{\circ} \mathrm{N}, 144^{\circ} \mathrm{E}$ (it is designated by a bold dagger) where the parameter $C$ has reached value 0.9622 .

To determine more exactly the obtained solution, we repeated the processing of initial data at a smaller search uncertainty $\left(41^{\circ}-43^{\circ} \mathrm{N}\right.$ and $\left.143^{\circ}-145^{\circ} \mathrm{E}\right)$ and a more exact search step $0.1^{\circ}$. The corresponding graph $C\left(\Phi_{e}, \Lambda_{e}\right)$ is shown on Fig. 8. The greatest value of the criterion parameter has made up $C_{\max }=0.9804$ at the point $\hat{\Phi}_{e}=41.7^{\circ} \mathrm{N}$, $\hat{\Lambda}_{e}=144.2^{\circ} \mathrm{E}$ (it is shown by a dagger). The coordinates of this point we have accepted as the estimations of the coordinates of the ionospheric perturbation observed during the Hokkaido earthquake.

For the Honshu earthquake, the graph $C\left(\Phi_{e}, \Lambda_{e}\right)$ (at $V=$ $\hat{V}$ and $H_{e}=\hat{H}_{e}$ ), constructed at a primary uncertainty of the search $\left(30^{\circ}-36^{\circ} \mathrm{N}\right.$ and $\left.134^{\circ}-140^{\circ} \mathrm{E}\right)$, and the value of the search step $0.1^{\circ}$ are shown in Fig. 9. The position of the source of ionospheric perturbation correspond to the point $\hat{\Phi}_{e}=33.0^{\circ} \mathrm{N}, \hat{\Lambda}_{e}=137.0^{\circ} \mathrm{E}$ of the maximum of the criterion parameter $C_{\max }=0.8371$. By analogy to Fig. 8 this point is also marked by a dagger in Fig. 9. An asterisk shows the earthquake epicenter position.

To illustrate convergence of the search process of an estimation of parameters $H_{e}$ and $V$ for the first experiment we show the graphs of the functions $C(V)$ (at $\Phi_{e}=\hat{\Phi}_{e}$, $\left.\Lambda_{e}=\hat{\Lambda}_{e}, H_{e}=\hat{H}_{e}\right)$ and $C\left(H_{e}\right)\left(\right.$ at $\Phi_{e}=\hat{\Phi}_{e}, \Lambda_{e}=\hat{\Lambda}_{e}$, $V=\hat{V})$ in Figs. 10 and 11 , respectively. One can see from Fig. 10 that the function $C(V)$ represents a combination of

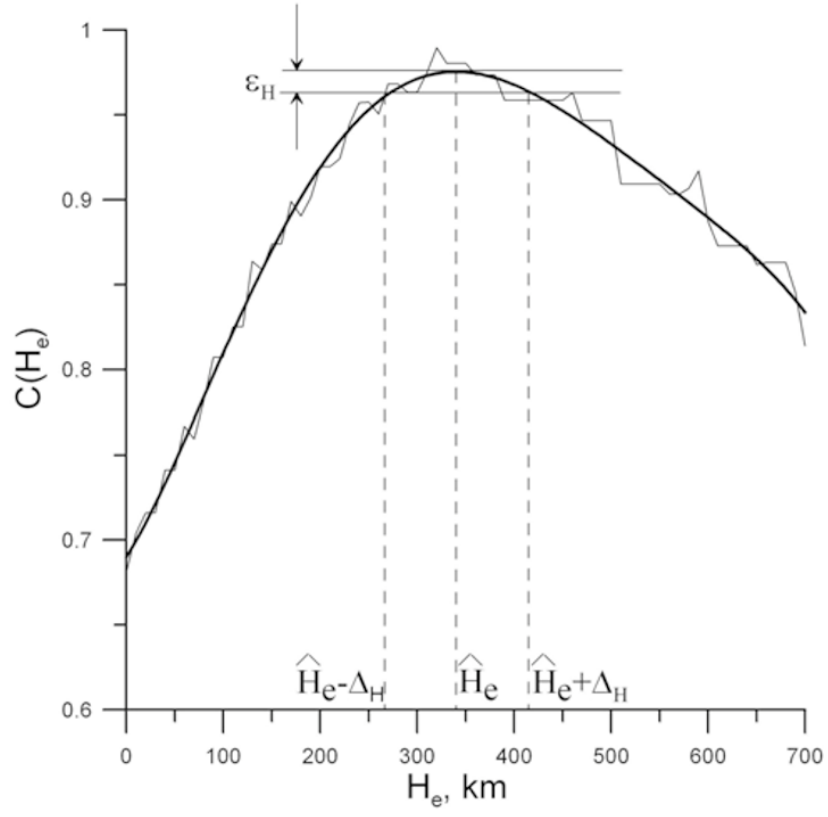

Fig. 11. The graph of the criterion parameter dependence on the height of the source of the ionospheric perturbation $C\left(H_{e}\right)$ for the Hokkaido earthquake. A bold curve shows the low-frequency "signal" component $C_{s}\left(H_{e}\right)$ obtained by polynomial approximation of $C\left(H_{e}\right)$. $\hat{H}_{e}=340 \mathrm{~km}$ is estimation of parameter $H_{e}$ at which the maximum of the functions $C_{s}\left(H_{e}\right)$ was reached. $\epsilon_{H}=0.02$ is an amplitude of the "noise" component $C_{n}\left(H_{e}\right)$ of dependence $C\left(H_{e}\right)$. The bounds of confidential interval of the estimation $\Delta_{H}= \pm 80 \mathrm{~km}$ also evaluation value $\hat{H}_{e}$ are marked by a dashed lines.

a low-frequency "signal" component $C_{S}(V)$ (it is marked by a bold curve) and a high-frequency "noise" component $C_{n}(V)$. The "signal" component was obtained by polynomial approximation of the initial function $C(V)$. The presence of the "noise" component $C_{n}(V)$ is the cause of an error occurrence at the estimation of parameter $V$ with using a method of maximum credibility. Therefore, as the estimation of parameter $V$ we considered such value at which the maximum of "signal" component $C_{s}(V)$ was reached.

In the experiment for Hokkaido earthquake, the maximum of the function $C_{s}(V)$ had reached at $\hat{V}=820 \mathrm{~m} / \mathrm{s}$. An estimation accuracy depends on curvature of "signal" function in the evaluation point and an amplitude of the "noise" component. To determine the confidence interval of the obtained estimation, we have drawn a line parallel to the axis $V$ on the level $\epsilon_{V}=0.02$ below the maximum of the "signal" function $C_{s}(V)$, where $\epsilon_{V}$ is an amplitude of the "noise" component $C_{n}(V)$. The points of crossing the drawn line and the $C_{S}(V)$ graph define the bounds of the estimation confidence interval. In the first experiment the confidence interval of an estimation of the perturbation propagation velocity reached $\Delta_{V}= \pm 60 \mathrm{~m} / \mathrm{s}$.

Similar reasoning and operations were carried out for the estimation of a height of the source $\hat{H}_{e}$ (Fig. 11). The maximal value of the "signal" component $C_{s}\left(H_{e}\right)$ reached $\hat{H}_{e}=340 \mathrm{~km}$. The value of the confidence interval of an estimation of the height was $\Delta_{H}= \pm 80 \mathrm{~km}$ at an amplitude of the "noise" component $\epsilon_{H}=0.02$. In Figs. 10 and 11 the bounds of the confidence intervals $\pm \Delta_{V}$ and $\pm \Delta_{H}$ as well as the evaluation values of the parameters $\hat{H}_{e}$ and $\hat{V}$ are 


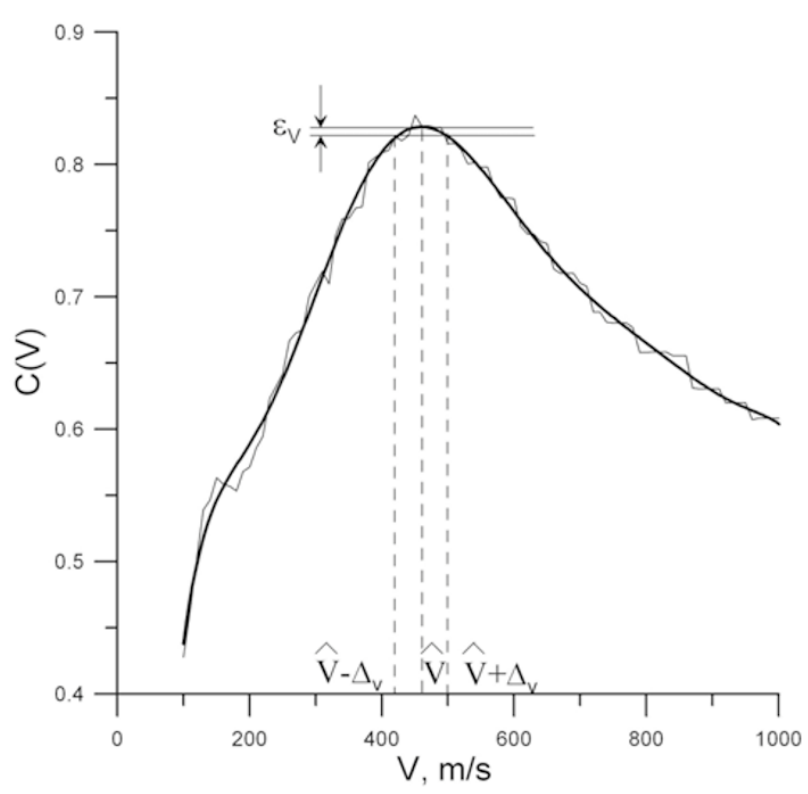

Fig. 12. Same as in Fig. 10, but for the Honshu earthquake. The velocity value $\hat{V}=460 \mathrm{~m} / \mathrm{s}$ is estimation of parameter $V . \Delta_{V}= \pm 40 \mathrm{~m} / \mathrm{s}$ is a confidential interval of estimation.

marked by dashed lines.

For the Honshu earthquake, we show the corresponding graphs $C(V)\left(\right.$ at $\left.\Phi_{e}=\hat{\Phi}_{e}, \Lambda_{e}=\hat{\Lambda}_{e}, H_{e}=\hat{H}_{e}\right)$ and $C\left(H_{e}\right)$ (at $\Phi_{e}=\hat{\Phi}_{e}, \Lambda_{e}=\hat{\Lambda}_{e}, V=\hat{V}$ ) in Figs. 12 and 13 , respectively. One can see from Fig. 12 that the maximal value of the "signal" component $C_{s}(V)$ has been reached at $\hat{V}=460 \mathrm{~m} / \mathrm{s}$. At that value, the accuracy of estimation of the perturbation propagation velocity reached $\Delta_{V}= \pm 40 \mathrm{~m} / \mathrm{s}$. The analysis of Fig. 13 shows that the evaluation value of the height of ionospheric perturbation source $\hat{H}_{e}=370 \mathrm{~km}$ corresponds to the height of maximum of ionospheric ionization but that the confidence interval of the height estimation is considerably larger than corresponding parameter for Hokkaido earthquake and reached $\Delta_{H}= \pm 130 \mathrm{~km}$. However, for all that, the amplitude of the "noise" component is less than in the first experiment and has reached $\epsilon_{H}=0.005$.

\section{Conclusion}

In this paper we present the quasi-optimum algorithm of spatiotemporal processing of TEC measurements of GPS array. The quasi-optimum algorithm allows the propagation velocity of coseismic ionospheric perturbation to be determined as well as the coordinates, height, and "switch-on" time of a source of perturbation without prior information about the disturbance form and site and time of the main shock of earthquake. The quasi-optimum algorithm realizes a coherent summation of TEC series reasoning from the maximization of energy of total signal of the ionospheric response.

We used the quasi-optimum algorithm for determining the parameters of the ionospheric perturbations which accompanied the earthquake in the vicinity of Hokkaido Island on September 25, 2003 and the earthquake near the south coast of Honshu Island on September 5, 2004. The results of these experiments show the high accuracy of

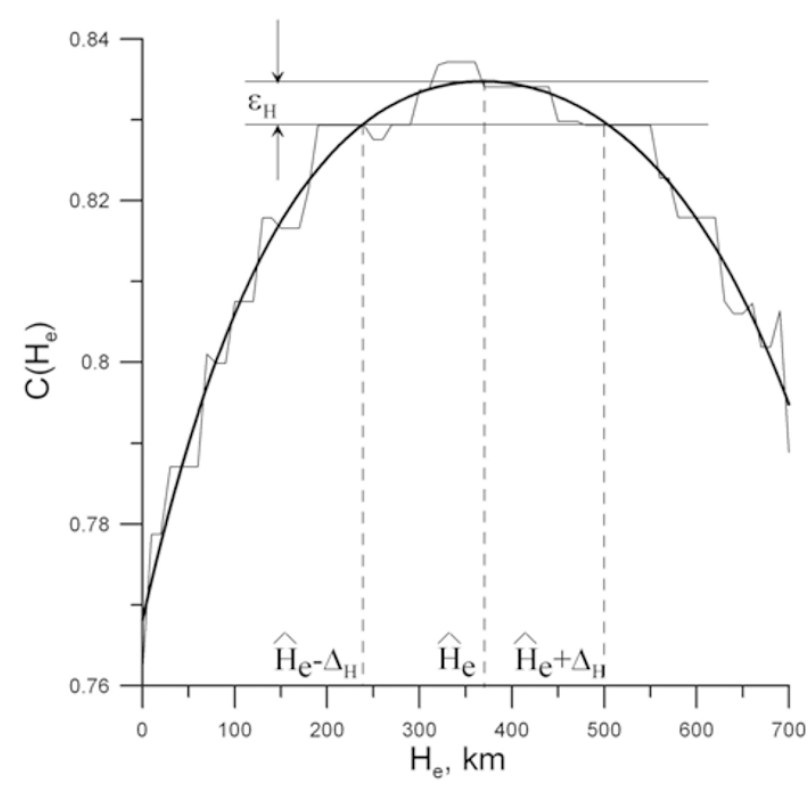

Fig. 13. Same as in Fig. 11, but for the Honshu earthquake. The velocity value $\hat{H}_{e}=370 \mathrm{~km}$ is estimation of parameter $H_{e} . \Delta_{H}= \pm 130 \mathrm{~km}$ is a confidential interval of estimation.

the perturbation source coordinates estimation $(33 \mathrm{~km}$ and $27 \mathrm{~km}$, respectively, with reference of the earthquakes epicenters).

The estimations of the perturbations propagation velocity $(820 \pm 60 \mathrm{~m} / \mathrm{s}$ and $460 \pm 40 \mathrm{~m} / \mathrm{s})$, heights $(340 \pm 80 \mathrm{~km}$ and $370 \pm 130 \mathrm{~km}$ ), and "switch-on" delay ( $346 \mathrm{~s}$ and $507 \mathrm{~s}$ ) of a source of the perturbation obtained in both experiments are in agreement with a theory according to which coseismic atmospheric disturbance propagates within a narrow cone of zenith angles up to ionospheric heights and then diverges in the form of a spherical wave with the radial velocity close to the speed of sound at these heights (Francis, 1975; Hocke and Schlegel, 1996; Afraimovich et al., 2001b; Rudenko and Uralov, 1995).

In both cases the shape of a front of the experimentally observed disturbances caused by the earthquakes is approximated to spherical wave with high conformity degree (0.9804 and 0.8371 respectively).

The parameter values which we have obtained for Hokkaido earthquake with the quasi-optimum algorithm of spatiotemporal processing of TEC measurements of GPS array agree with the results of earlier researches of ionospheric perturbation caused by this earthquakes (Afraimovich et al., 2005, 2006; Heki and Ping, 2005).

The difference in velocity values obtained in these experiments may be explained by the different conditions of perturbation propagation in each experiment, caused by different local time (06 LT for Hokkaido earthquake and $00 \mathrm{LT}$ for Honhsu earthquake) and, consequently, by different values of the electrons temperature (Roble, 1975).

Acknowledgments. Authors are grateful to S. V. Voeykov for the help in primary data processing and E. I. Astafyeva for the earthquake information preparation. The authors wish to thank members of Scripps Orbit and Permanent Array Center (SOPAC) for the RINEX files used in this paper. The work was supported by the SB RAS collaboration project N 3.24 and RFBR grants 05-05- 
64634 and 06-05-64577. Authors are also grateful to two referees for their contribution in improving of paper.

\section{References}

Afraimovich, E. L., GPS global detection of the ionospheric response to solar flares, Radio Sci., 35(6), 1417-1424, 2000.

Afraimovich, E. L., E. A. Kosogorov, N. P. Perevalova, and A. V. Plotnikov, The use of GPS array in detecting shock-acoustic wave generated during rocket launchings, J. Atmos. Solar-Terr. Phys., 63, 1941-1957, 2001a.

Afraimovich, E. L., N. P. Perevalova, A. V. Plotnikov, and A. M. Uralov, The shock-acoustic waves generated by the earthquakes, Ann. Geophys., 19, 395-409, 2001b.

Afraimovich, E. L., V. V. Chernuhov, and V. V. Kiryushkin, SpatialTemporal Characteristics of the Ionospheric Disturbance Induced by Shock Acoustic Waves Due to Rocket Launching, J. Commun. Technol. Electron., 46, 1199-1206, 2001c.

Afraimovich, E. L., V. V. Kiryushkin, and N. P. Perevalova, Determination of the Characteristics of Ionospheric Perturbations in the Near-Field Region of an Earthquake Epicenter, J. Commun. Technol. Electron., 47, 739-747, 2002.

Afraimovich, E. L., E. I. Astaf'eva, and V. V. Kiryushkin, Ionospheric Disturbance in the Near-Region of an Earthquake Epicenter on 25 September 2003, Radiophys. Quantum Electron., 48, 299-313, 2005.

Afraimovich, E. L., E. I. Astaf'eva, and V. V. Kiryushkin, Localization of the source of ionospheric disturbance generated during an earthquake, Int. J. Geomagnet. Aeronomy, 6, GI2002, doi:10.1029/2004GI000092, 2006.

Calais, E. and J. B. Minster, GPS detection of ionospheric perturbations following the January 1994, Northridge earthquake, Geophys. Res. Lett., 22, 1045-1048, 1995.

Calais, E. and J. B. Minster, GPS detection of ionospheric perturbations following Space Shuttle assent, Geophys. Res. Lett., 23, 1897-1900, 1996.

Calais, E., J. B. Minster, M. A. Hofton, and M. A. H. Hedlin, Ionospheric signature of surface mine blasts from Global Positioning System measurements, Geophys. J. Int., 132, 191-202, 1998.

Calais, E., J. S. Haase, and J. B. Minster, Detection of ionospheric perturbations using a dense GPS array in Southern California, Geophys. Res. Lett., 30, 1628-1631, 2003.

Fitzgerald, T. J., Observations of total electron content perturbations in GPS signals caused by a ground level explosion, J. Atmos. Solar-Terr. Phys., 59, 829-834, 1997.

Francis, S. N., Global propagation of atmospheric gravity waves: A review, J. Atmos. Terr. Phys., 37, 1011-1039, 1975.

Heki, K. and J. Ping, Directiivity and apparent velocity of the coseismic ionospheric disturbances observed with a dense GPS array, Earth Planet. Sci. Lett., 236, 845-855, 2005.

Hocke, K. and K. Schlegel, A review of atmospheric gravity waves and travelling ionospheric disturbances: 1982-1995, Ann. Geophys., 14, 917-940, 1996.

Montes, H. A. and E. S. Posmentier, Co-phase analysis of atmospheric wave data, Geophys. J. R. Astr. Soc., 26, 271-278, 1971.

Roble, R. G., The calculated and observed diurnal variation of the ionosphere over Millstone Hill on 23-24 March 1970, Planet. and Space Sci., 23, 1017-1033, 1975.

Rudenko, G. V. and A. M. Uralov, Calculation of ionospheric effects due to acoustic radiation from an underground nuclear explosion, J. Atmos. Terr. Phys., 57, 225-236, 1995.

E. L. Afraimovich (e-mail: afra@iszf.irk.ru) and V. V. Kiryushkin 